\title{
A hypothesis for understanding mechanisms of normal and abnormal behavior states based on operation hypothesis
}

\begin{abstract}
To express appropriate context-dependent behavior, functional linkage between the forebrain and hindbrain should be necessary. We propose that the functional linkage occurs at the level of the midbrain according to the "Operation Theory" advocated by Gerald Edelmann.1 During wakefulness, signals from the neocortex and limbic system evoke volitional and emotional behaviors, respectively. On the other hand, activity of limbic system elicits rapid eye movement (REM) sleep accompanied by muscular atonia. These state-dependent motor functions can be achieved by appropriate functional linkage between systems existing in forebrain and hindbrain. However, the functional linkage collapses in some neurological disorders such as REM sleep behavior disorders and narcolepsy. Here, attempts are made to understand the pathophysiology of these neurological disorders and to elucidate the mechanisms of motor function depending on awake-sleep states. Roles played by neurotransmitter systems and by the basal ganglia are further considered in relation to the excitability modification of the forebrain and hindbrain structures.
\end{abstract}

Keywords: psychological states, wakefulness, REM sleep, neurotransmitters, brain
Volume 2 Issue I - 2018

\author{
Kaoru Takakusaki,' Mirai Takahashi,' Toshi \\ Nakajima,' Ryosuke Chiba,' Kazuhiro \\ Obara, 'Tsukasa Nozu, ${ }^{2}$ Toshikatsu \\ Okumura $^{3}$ \\ 'Research Center for Brain function and Medical Engineering, \\ Asahikawa Medical University, Japan \\ 2Department of Regional Medicine and Education, Asahikawa \\ Medical University, Japan \\ ${ }^{3}$ Department of Internal Medicine,Asahikawa Medical University, \\ Japan
}

\author{
Correspondence: Kaoru Takakusaki, MD PhD, Professor and \\ Director, Research Center for Brain Function and Medical \\ Engineering, Asahikawa Medical University, Midorigaoka-Higashi \\ 2-I, I-I,Asahikawa 078-85 I I, Japan, Tel +8I-I66-68-2884, Fax \\ +8I-166-68-2887, Email kusaki@asahikawa-med.ac.jp
}

Received: January 10, 2018 | Published: January 29, 2018

Abbreviations: AMD, amygdale; ARAS, ascending reticular activation system; BF, basal forebrain; CLR, cerebellar locomotor region; $\mathrm{CNF}$, cuneiform nucleus; $\mathrm{CPG}$, central pattern generators; CTX, cortex; DA, dopamine; E, extensor motoneurons; F, flexor motoneurons; GABA, gamma-amino butyric acid; GPi, internal segment of globus pallidus; Hipp, hippocampus; LC, locus coeruleus; LHA, lateral hypothalamic area; MLR, midbrain locomotor region; NAc, nucleus accumbens; NRGc, nucleus reticularis gigantocellularis; NRMc, nucleus reticularis magnocellularis; ORX, orexin; PD, parkinson's disease; PM, premotor area; PMRF, pontomedullary reticular formation; PPN, pedunculopontine tegmental nucleus; PRF, pontine reticular formation; RN, raphe nuclei; SLR, subthalamic locomotor region; SMA, supplementary motor area; SNc, substantia nigra pars compacta; REM, rapid eye movement; $\mathrm{SNr}$, substantia nigra reticulate; STN, subthalamic nucleus; Thal, thalamus; VP, ventral pallidum; VST, vestibulo spinal tract; VTA, ventral tegmental area

\section{Introduction}

Despite the electroencephalographic similarities, the psychological states of wakefulness and REM sleep are obviously different. The neocortex is active during wakefulness, while the sub cortical structures including the limbic areas and basal ganglia are more active than the neocortex during rapid eye movement (REM) sleep. ${ }^{2,3}$ The difference in the psychological condition is associated with different motor functions depending on behavioral states (awake-sleep states). Under explicit consciousness condition during wakefulness, purposeful goal-directed behaviors are initiated by signals arising from either volitional processing in the cerebral cortex or emotional processing in the limbic system. Regardless of whether the locomotion is volitional or emotional, it is accompanied by automatically controlled movement processes such as the adjustment of postural muscle tone and rhythmic limb movements. ${ }^{4,5}$ On the other hand, muscle tone is completely abolished during REM sleep even if the limbic system is more active. However, these relationships between the psychological states and the motor functions collapse in specific neurological disorders. Patients with REM sleep behavior disorders (RBD) exhibit abnormal behaviors that are probably based on dream experience or emotional memory. ${ }^{6}$ On the other hand, strong emotional stimulus suddenly triggers muscular atonia that resembles REM sleep (cataplexy) in narcolepsy patients. ${ }^{7}$ However, the patients maintain explicit conscious state during cataplexy. ${ }^{8}$

While the forebrain, including the cerebral cortex (neocortex) and limbic systems, play major role to produce and maintain psychological states depending on behavioral context, neural constituents involved in the execution of basic motor functions such as regulation of postural muscle tone and initiation of locomotion exist in the hindbrain including the brainstem and spinal cord.,5,9 Then, what kind of mechanism does link neural systems existing in the forebrain and the hindbrain so that appropriate behaviors could be produced depending on awake-sleep states? In addition, are pathological conditions such as RBD and narcolepsy induced by selection mistakes between systems in the forebrain and hindbrain? Elucidation of these issues not only leads to an understanding the mechanisms of normal awake-sleep states but also contributes to the understanding the pathophysiological mechanisms of these neurological disorders.

To answer these questions, we propose a "gating mechanism at the level of the midbrain" that enables functional linkage between 
selected systems in each of the forebrain and hindbrain according to the "Operation Theory" which was advocated by G Edelmann. ${ }^{1}$ Specifically, a system exhibiting dominant activity ("excitability preference") is selected in the forebrain and hindbrain, and the selected systems are functionally linked at the midbrain ("gate"), whereby appropriate motor functions or behaviors can be achieved depending on behavioral state (awake-sleep state) and behavioral context. Neurotransmitters such as monoamines, acetylcholine and orexin are particularly important to determine the excitability preference of the target systems. Because these transmitter systems exhibit state-dependent alteration, ${ }^{10-13}$ they modulate the excitability of the forebrain and hindbrain structures through their extensive projections. Moreover, it deserves considering the role of the basal ganglia in relation to the determination of the excitability preference and selection of the target systems. ${ }^{14}$ While the midbrain receives excitatory inputs from the cerebral cortex and limbic system, it also receives inhibitory inputs from the basal ganglia. ${ }^{4,5}$ In addition, emotional signals in the limbic-hypothalamus and volitional signals in the cerebral cortex are modulated by the output of the basal ganglia. Basal ganglia efferents to both the forebrain and the midbrain are therefore critical in the selection of either volitionally-initiated movements or emotionally-triggered behaviors.

Because forebrain structures drive motor systems in the hindbrain structures to produce various types of behaviors, we will first outline basic forebrain and hindbrain structures involved in the control of postural muscle tone and locomotion. Then, we will consider the mechanisms by which the systems existing in forebrain and hindbrain are functionally connected in relation to the behavioral state control. Finally, we will discuss the pathophysiological mechanisms of sleeprelated movement disorders including those observed in Parkinson's disease (PD).

\section{Forebrain structures}

\section{Two major systems in the forebrain}

Figure 1 summarizes our knowledge of neuronal mechanisms of cognitive and emotional control of locomotion in the cat based on previous studies. ${ }^{15-20}$ There are two major systems in the forebrain. Here we define "dorsal forebrain system" and "ventral forebrain system" as a matter of convenience. The former constitutes neocortexneostriatum-dorsal pallidal pathway (Figure 1A) and, the latter, which is an older part of the forebrain structures in view of the vertebrate evolution, ${ }^{9,21}$ constitutes archicortex-ventral striatum-ventral pallidal pathway (Figure 1B). In addition, the ventral and dorsal pallidum also project back to groups of cells within the thalamus which, in turn, project back to the cortex. ${ }^{22}$

In the ventral forebrain system (Figure 1B), emotional signals arising from the amygdala via the subthalamic locomotor region (SLR), which exist in the lateral hypothalamic area (LHA), exert excitatory effects upon brainstem motor centers including the mesencephalic locomotor region (MLR) and posture control region in the pedunculopontine tegmental nucleus (PPN) ${ }^{23}$ On the other hand, the nucleus accumbens (ACC), as a component of the limbic system, may be important in releasing locomotion, via gamma-Aminobutyric acid (GABA)-ergic projections dis-inhibiting the MLR via the ventral pallidum ${ }^{24,25}$ and via fibers to the substantia nigra pars reticulate (SNr). ${ }^{26}$ Because the ACC receives inputs from the hippocampus and amygdala, the ventral system may be implicated in reward-oriented and emotionally-triggered behaviors, ${ }^{21}$ as it receives dopamine (DA) inputs from the ventral tegmental area (VTA).

The more recently evolved parts of the basal ganglia make up the dorsal pathway (Figure 1A). ${ }^{9}$ Neostriatum-dorsal pallidal pathway exerts GABAergic inhibitory effects upon brainstem motor centers via the SNr. Cortico-fugal projections to the brainstem and spinal cord are excitatory output from the dorsal system. Cortico-fugal projections to the pontomedullary reticular formation (PMRF) arise from the pre motor cortices (SMA/PM) in quadruped ${ }^{27}$ and biped ${ }^{28}$ animals. Probabilistic diffusion tractography in rhesus monkey as well as humans shows that the SMA is strongly connected to the lateral PPN, while the dorsal PM is connected to the medial PPN. ${ }^{29}$

In the higher primate with bipedal gait, SMA/PM projections to the PPN and PMRF may play a crucial role of initiation of locomotion with appropriate postural control. ${ }^{30}$ As these motor cortical areas receive sensory and cognitive information from other cortical areas, ${ }^{31}$ dorsal forebrain system may achieve precise movements depending on cognitive behavioral context, such as sensory-guided and volitionallyinitiated locomotor control. ${ }^{4}$

\section{Role of the basal ganglia}

The output of the basal ganglia consists of tonically active GABAergic neurons originating from two nuclei, globus, pallidus, interna (GPi) and SNr. Subpopulations of GABAergic output neurons from both structures have prominent projections to different brainstem centers (hindbrain) and another part projecting to thalamus and back to cortex (forebrain).

One important role of the basal ganglia output is to keep the different motor centers in the brainstem as well as different programs in the cerebral cortex under inhibitory control during resting conditions, so that they are released when intended. At the resting condition, tonic $\mathrm{GPi} / \mathrm{SNr}$ activity maintains a continuous inhibitory drive to different motor centers in the brainstem, such as the superior colliculus (SC), MLR, postural centers, swallowing central pattern generators (CPGs). 4,9,14,32,33

A dis-inhibition of thalamocortical neurons caused by a cessation of $\mathrm{GPi} / \mathrm{SNr}$ discharge is thought to increase the level of excitation of the different cortical target areas. Triggering a motor program will depend on removal of this tonic inhibition and therefore the pallidal output neurons in turn need to be inhibited from the input layer of the basal ganglia, the striatum. ${ }^{14,21,25,32} \mathrm{~A}$ prerequisite for activating or releasing the activity of the target system is that this GABAergic inhibition is temporarily reduced. This can be achieved through activation of GABAergic projection neurons from striatum given an appropriate synaptic drive from cortex, thalamus and the DA system. ${ }^{14,21}$ The neostriatum and ventral striatum receive dopaminergic inputs from the SNc and VTA, ${ }^{26}$ respectively. In PD, DA neurons in both areas are severely damaged, ${ }^{34}$ indicating that functions of the dorsal and ventral forebrain systems are seriously disturbed in this disease.

\section{Motor systems in the hindbrain}

In the brainstem there are motor centers involved in various types of pattern movements, such as respiration, eye movements, micturition, swallowing, mastication, locomotion and postural control, etc.. ${ }^{9,32-}$ 33 To effectively develop the subsequent consideration, here we 
particularly focus on the mechanism of controlling postural muscle tone and locomotion.

\section{Muscle tone control systems}

Muscle tone excitatory system: Noradrenaline (NA) neurons of the locus coeruleus (LC) and serotonin (5-HT) neurons of the raphe nuclei $(\mathrm{RN})$ are involved in maintenance of arousal level as components of the ascending reticular activation system (ARAS). ${ }^{13}$ On the other hand, these neurons also contribute to maintenance of postural muscle tone during wakefulness via coerulospinal and raphespinal tracts. Because an activation of neurons in these nuclei increased the excitability of the motoneuron in the spinal cord, resulting in an increase in the level of muscle tone, these mono aminergic descending tracts are considered

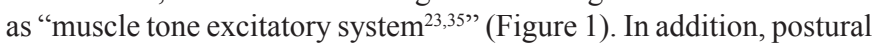
muscle tone is also increased by stimulating the ventral part of the medullary reticular formation (MRF) corresponding to the nucleus reticularis magnocellularis (NRMc), ${ }^{36}$ indicating that excitatory reticulospinal pathway arising from this region also operates as muscle tone excitatory system. Because neurons in the LC and RN project to the NRMc, the excitatory reticulospinal system cooperates with monoaminergic descending tracts to maintain and increase the level of postural muscle tone. The vestibulospinal tract also maintains postural balance in response to changes in sense of equilibrium.

Orexin (ORX) neurons in the LHA exhibit state-dependent alteration with high firing rates during wakefulness and low rates during REM sleep. ORX neurons project to the LC and RN and modulate the excitability of monoamine neurons. ${ }^{36,37}$ It has been also suggested that the ORX system contributes to maintenance of postural muscle tone via excitatory reticulospinal tract. ${ }^{38}$ Accordingly, ORX system acts on the muscle tone excitatory systems so that postural muscle tone is appropriately regulated in accordance with awakesleep states. Conversely, damages to ORX neurons may disturb the excitability muscle tone excitatory system during wakefulness. ${ }^{35,39}$

Muscle tone inhibitory system: Acetylcholine (ACh) neurons in the PPN have descending projection to the PMRF in addition to ascending projections to the non-specific thalamic nuclei and midbrain DA neurons in the SNc and VTA (Figure 1). The descending cholinergic system activates "muscle tone inhibitory system" which is composed by rostral pontine reticular formation (PRF) neurons, reticulospinal neurons in the MRF corresponding to the nucleus reticularis gigantocellularis (NRGc) and spinal inhibitory inter neurons ${ }^{18,40-42}$ (Figure 1). The muscle tone inhibitory system may therefore regulate locomotion in addition to postural muscle tone of whole body via its widespread branching to spinal segments of whole neuraxis. The inhibitory inter neurons in this system provide not only postsynaptic inhibitory effects upon motoneurons and inter neurons mediating reflex pathways but pre synaptic inhibitory effects upon sensory afferents, ${ }^{40-42}$ resulting in suppression of postural muscle tone, spinal reflexes and activity of CPG. The inhibitory actions possibly use glycine and GABA.

Because an activation of PPN-ACh induces REM and muscular atonia, the muscle tone inhibitory system contributes to the generation of REM sleep. ${ }^{41,44}$ While firing rate of ACh neurons is higher in wakefulness and REM sleep, ${ }^{10,13}$ wakefulness-relating neurons such as ORX neurons and monoamine neurons stop firing during REM sleep. Therefore, atonia during REM sleep is due to higher activity in the inhibitory system and lower activity in the excitatory system. GABAergic neurons in the PPN and those in the SNr inhibit PPN-
ACh neurons, ${ }^{44,45}$ indicating that GABAergic neurons modulate REM sleep by acting on PPN-ACh neurons. ${ }^{35}$ The ORX system during wakefulness inhibits PPN-ACh neurons by activating these GABAergic neurons. ${ }^{35}$
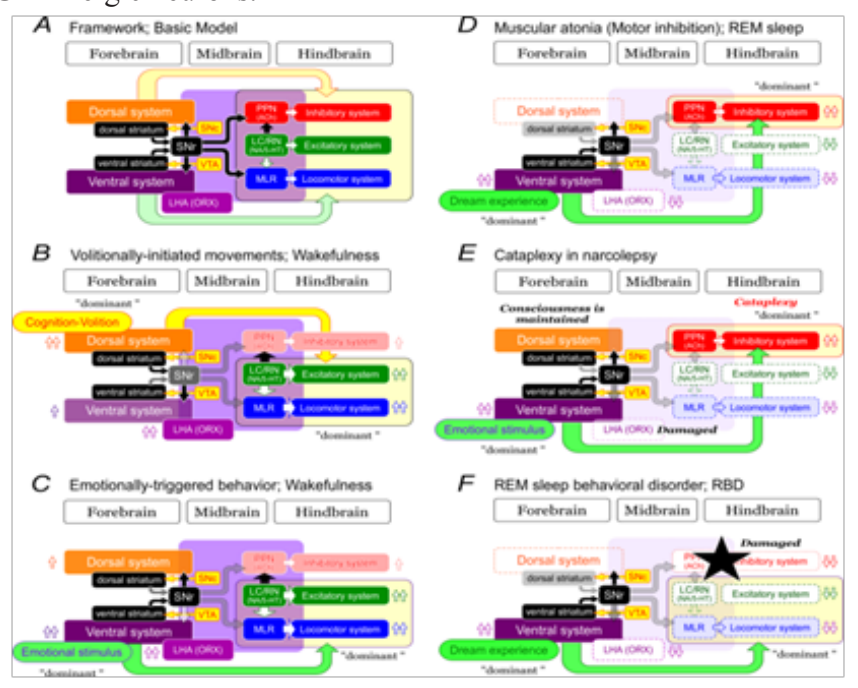

Figure I Neuronal mechanisms of cognitive (A) and emotional (B) control of locomotion in the cat.

(A) Dorsal forebrain system for cognitive locomotor control. A visuo-motor pathway from the visual cortex to motor cortex via the parietal cortex contributes to this control. Corticofugal projections act on to the basal ganglia nuclei, brainstem and spinal cord. Dopaminergic projection from the substantia nigra pars compacta $(\mathrm{SNc})$ to the caudate-putamen $(\mathrm{CPu})$ may be involved in learning the locomotor behaviors. GABAergic output from the basal ganglia nuclei (internal segment of the globus pallidus and substantia nigra pars reticulata; GPi/SNr) acts on MLR/PPN area and may control locomotion and posture. Efferents from the midbrain locomotor region (MLR) recruit excitatory system, inhibitory system and locomotor pathway. The excitatory system arises from the locus coeruleus (LC) and the raphe nuclei (RN). The inhibitory system which arises from cholinergic neurons in the pedunculopontine tegmental nucleus (PPN) sequentially activates pontine reticular formation (PRF) neurons, medullary reticulospinal neurons in the nucleus reticularis gigantocellularis (NRGc) and spinal inhibitory interneurons. The inhibitory interneurons may inhibit both motoneurons and interneurons. The locomotor pathway consists of reticulospinal neurons arising from the ventromedial medulla corresponding to the nucleus reticularis magnocellularis (NRMc). Cholinergic and glutamatergic projections from the PPN excite SNc-DA neurons. These descending tracts act on central pattern generators (CPGs) in spinal cord so that muscle tone and locomotion are regulated. Efferents from the cerebellar locomotor region (CLR) may excite locomotor pathway.

(B) Ventral forebrain system for emotional locomotor control. Efferents from the amygdala (AMD) and hippocampus (Hipp) project to the nucleus accumbens (NAc). GABAergic NAc neurons project to ventral pallidum (VP) and the SNr, which control activity of the MLR/PPN neurons. Efferents from the AMD and the Hipp also act on lateral hypothalamic area, which corresponds to the SLR. DA projections from the ventral tegmental area (VTA) may contribute to the reward-oriented locomotor behaviors. Abbreviations: $\mathrm{E}$; extensor motoneurons, F; flexor motoneurons. Modified from Figure 4 in ref. 20. Takakusaki K (2017).

Mutual interaction between the muscle tone control systems: There is a "mutual inhibitory interaction" between the muscle tone excitatory system and inhibitory system. Monoaminergic neurons projecting to the PRF inactivate the muscle tone inhibitory system. ${ }^{46,47}$ In addition, monoaminergic projection to the PPN also inhibits mesopontine ACh neurons. ${ }^{48}$ On the other hand, GABAergic neurons 
in the PMRF which constitute the inhibitory system act on neurons in the LC and inhibit the excitatory system. ${ }^{49}$ Therefore, postural muscle tone in awake-sleep states is regulated by the mutual inhibitory interaction of these systems, and the breakdown of the interaction may disturb muscle tone.

\section{Locomotor system}

Three locomotor regions are identified. They are the MLR, the SLR and the cerebellar locomotor region (CLR) $)^{5,19,20}$ (Figure 1). An imaging study revealed that these regions are also found in human. ${ }^{50}$ The MLR mostly corresponds to the cuneiform nucleus (CNF) and dorsal part of the PPN. ${ }^{36,45}$ Signals from the MLR may activate "muscle tone excitatory system" and "locomotor system". The locomotor system is composed of the excitatory reticulospinal tract arising from the NRMc and CPG in the spinal cord. The CLR corresponds to the mid-part of cerebellar white matter which contains massive fibers connecting bilateral fastigial nuclei. ${ }^{51}$ Signals in the CLR are thought to drive the vestibulospinal tract and reticulospinal tract. ${ }^{52}$

The SLR corresponds to the LHA where ORX neurons are present. Emotional signals through the ventral forebrain system are expected to activate the SLR, which, in turn, activates the locomotor system either directly or indirectly via the MLR. ${ }^{9,15,53}$ ORX acts on the MLR and elicits locomotion. ${ }^{35}$ Considering that the SLR mostly corresponds to the origin of the ORX system, both are involved in emotional motor behaviors including locomotion and eating. ${ }^{37}$

\section{Basal ganglia projections to the midbrain}

GABAergic projection from the $\mathrm{SNr}$ to the midbrain regulates locomotion and postural muscle tone. ${ }^{44,45}$ GABAergic projections from the lateral and medial SNr to the PPN and MLR reduce the excitabilities of the muscle tone inhibitory system and locomotor system, respectively. This indicates that output of the basal ganglia to the PPN/MLR independently regulates muscle tone and locomotion. Excessive increase in the GABAergic basal ganglia output to the brainstem, as in the case in $\mathrm{PD}$, therefore, results in gait failure and hypertonus (muscular rigidity). The muscular rigidity is due to contractions of antigravity (extensor) muscles and flexor muscles (co-contraction).$^{54}$ Accordingly, basal ganglia efferents to the muscle tone control systems may contribute to regulate muscle co-contraction which enables to maintain stiffness of limb joints so that bodily equilibrium can be stabilized during ongoing movements in addition when starting and halting.

In $\mathrm{PD}$, deterioration of the $\mathrm{DA}$ neurons excessively increases GABAergic output from the basal ganglia. ${ }^{22}$ Gait akinesia, freezing and rigidity in this disease are assumed to be induced by the enhancement of the GABAergic basal ganglia efferents to the brainstem in addition to the thalamocortical neurons. ${ }^{22,55}$ GABAergic projections from the dorsolateral part of the $\mathrm{SNr}$ to the $\mathrm{SC}$ and to the swallowing center control saccadic eye movements ${ }^{32,56}$ and swallowing, ${ }^{57}$ respectively.

\section{Functional linkage between forebrain and hindbrain;} Integration of consciousness and behavior

Functional linkage between the forebrain and hindbrain during awake-sleep states: To consider functional linkage between the forebrain and hindbrain, neuronal structures illustrated in Figure 1 are simplified as shown in Figure 2A. There are dorsal and ventral forebrain systems, and muscle tone control systems and locomotor system in the hindbrain. The forebrain systems have excitatory and inhibitory projections to the hindbrain motor systems. While ORX neurons have extensive projections, the midbrain, which includes the $\mathrm{SNr}$ (GABA), SNc/VTA (DA), PPN (ACh), LC (NA), RN (5-HT) in addition to the MLR, is the major target. ORX directly excites these neurons. ${ }^{37}$ However, it inhibits PPN-ACh neurons via GABAergic neurons. ${ }^{35}$

\section{Wakefulness}

During wakefulness, excitability of neural systems in the forebrain and hindbrain is appropriately regulated by neurotransmitters. Because of the higher activity ORX neurons, monoamine (NA/5-HT) neurons are more active than PPN-ACh neurons, indicating that the excitability of muscle tone excitatory system and locomotor system is higher than that of inhibitory system. Therefore, an activation of forebrain structures exclusively activates excitatory and locomotor systems in the hindbrain. Namely, the dorsal forebrain system may produce "context-dependent volitionally-initiated movements" by acting on this hindbrain motor systems (Figure 2B). On the other hand, a strong emotional stimulus activates the ventral forebrain system, which, in turn, also excites the hindbrain systems so that "emotional-triggered behavior" is elicited (Figure 2C).

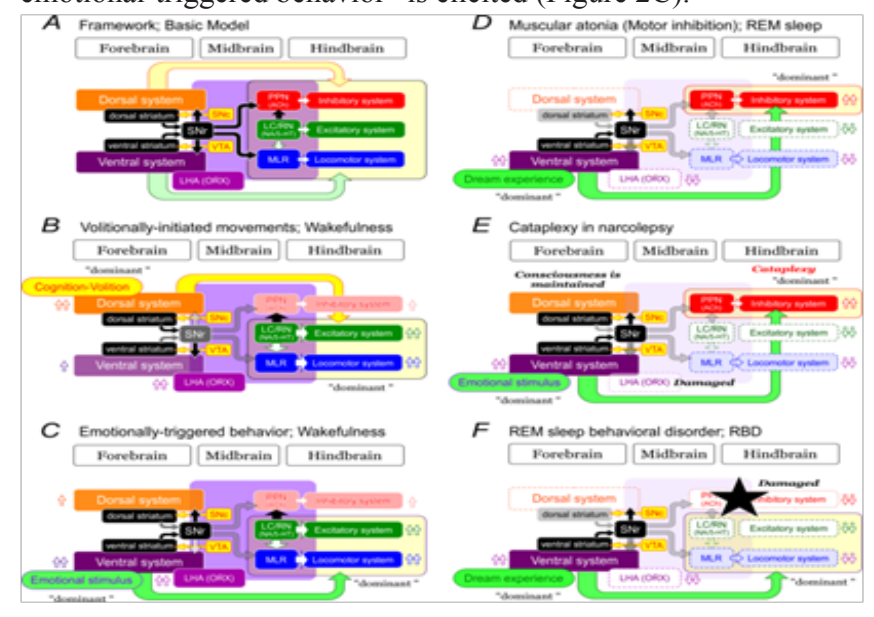

Figure 2 Hypothetical models of normal and abnormal behavior state control.

(A) Framework of the basic model

B to F: Mechanisms of volitionally-initiated movements during wakefulness.

(B) Emotionally-triggered behavior during wakefulness

(C) Muscular atonia (motor inhibition) during REM sleep

(D) Cataplexy in narcolepsy patients

(E) REM sleep behavior disorder

(F) See text for explanation.

\section{Slow-wave sleep}

During slow wave sleep (non-REM sleep) period, the activity of ORX neurons, ACh neurons and monoamine neurons is reduced. As a result, arousal level declines in accordance with reduced activity in the ARAS. Activity of muscle tone control systems is also reduced. Because of declined activity in the forebrain and hindbrain structures, functional linkage between these structures becomes weak.

\section{REM sleep}

When one enters REM sleep, firing of ORX neurons and monoamine neurons reduces, while the firing of ACh neurons in 
the PPN and the basal forebrain increases. Activation of these ACh neurons activates the ARAS and induces a low amplitude fast wave (de synchronization) resembling that seen in wakefulness (EEG arousal). Despite the electroencephalographic similarities, the psychological states of wakefulness and REM sleep are obviously different. The neocortex is less active, but the limbic areas are more active. ${ }^{2,3}$ Since the cholinergic PPN neurons excite DA neurons ${ }^{58}$ higher DA activity might be involved in the hyperactivity of the basal ganglia and limbic system. Higher ACh activity together with the low monoamine and ORX activities increases the excitability of muscle tone inhibitory system (Figure 2D). Because "dream experience" accompanying images and sounds coincides with the period when the REM appears, ${ }^{3}$ higher activity of the ventral forebrain system may preferentially activate the inhibitory system so that muscle tone is completely depressed during REM sleep (Figure 2D). Therefore, it is interesting that the activation of the limbic system that develops emotional behaviors during wakefulness is thought to induce generalized motor inhibition during REM sleep.

\section{Pathological conditions}

\section{Disturbance of orexinergic system pathophysiological mechanisms of narcolepsy}

and

In narcoleptic patients and animals, emotional signals elicit a sudden loss of muscle tone (cataplexy), which is caused by degeneration of orexin neurons in the LHA. ${ }^{7,59}$ Autoimmune process may be involved in the degeneration of ORX neurons.$^{60}$ Emotional stimulus during wakefulness usually induces emotional behaviors such as "fight or flight" reactions which accompany augmentation of muscle tone and locomotion (Figure 2C). However, cataplexy in narcolepsy patients is induced by elevation of emotion accompanied by laughter, joy and feeling that self-esteem is tickled. Possibly, cataplexy is triggered by neural mechanisms that induce REM sleep. ${ }^{7,61}$ During REM sleep our consciousness is not clear, because the activity of the neocortex is quite low while that of the limbic system and basal ganglia are higher. ${ }^{3}$ In contrast, cerebral cortex activity in narcolepsy patients is well preserved during the period of cataplexy, and awareness is maintained. ${ }^{7,61}$ Narcolepsy patients suffer from sudden onset of REM sleep (SOREM), because non-REM sleep is extremely short or lacked in this disease. This suggests that cataplexy is triggered by mechanisms different from the normal REM sleep.

Then, the question is how cataplexy is produced in narcolepsy patients? In the absence of ORX, the excitability of both the locomotor system and the muscle tone excitatory system would be greatly reduced. By contrast, the excitability of muscle tone inhibitory system would be elevated. If this is the case, emotional stimulus activate ventral forebrain system, which, in turn, selectively activate the inhibitory system so that cataplexy is evoked (Figure 2E). However, consciousness in narcolepsy patients is maintained even during cataplexy period. We propose that orexin may be a determinant for the selection of emotional motor behavior. Consequently, the excitability of the hindbrain motor systems is strongly modified depending on the presence or absence of ORX. As a result, identical emotional stimulus triggers opposite types of behaviors, "emotional behaviors during normal wakefulness" and "cataplexy in narcolepsy patients".

Excessive daytime sleepiness (EDS) also appears when narcolepsy patients have difficulties in maintaining arousal state because of reduced activity in the arousal systems. In mice with impaired orexinergic system, the activity of monoaminergic neurons becomes unstable. ${ }^{62}$ This may induce fragmentation of sleep and destabilization of non-REM sleep, resulting in the possibility of SOREM.

\section{REM sleep behavior disorders (RBD $\square$}

The mechanisms of REM sleep behavioral disorders (RBD) may suggest a role for a limbic control of locomotor behavior. RBD is a pathophysiological condition in which abnormal movements and behaviors are induced during REM sleep. ${ }^{6}$ Lesions in the PMRF of the cat show aggressive behaviors during REM sleep. ${ }^{63}$ This is called as "REM without atonia" which is an animal model of RBD. $\mathrm{RBD}$ in humans was induced by microinfarctions of the dorsolateral or medial part of the pons. ${ }^{6}$ This region approximately corresponds to the PPN and medial PRF, origin and relay nucleus of the muscle tone inhibitory system, respectively (Figure 1 ). There are evidences showing that RBD highly complicate with multiple system atrophy ${ }^{64}$ and progressive supranuclear palsy ${ }^{65}$ in which mesopontine ACh neurons are severely degenerated. Moreover, anti-cholinergic agents were effective in the treatment of the RBD. ${ }^{66}$ Therefore, damages in the mesopontine $\mathrm{ACh}$ neurons and dysfunction of the muscle tone inhibitory system may be involved in this syndrome. However, this alone cannot explain the RBD. Abnormal behavior reflects the content of the dream experience which associates with higher activity of the sub cortical structures during REM sleep. Because the excitability of the locomotor system as well as the muscle tone excitatory system is somewhat preserved due to either the less activity of ACh neurons or insufficient activity of the inhibitory system, emotional signals associating with dream experience during REM sleep preferentially select and activate muscle tone excitatory system and drive locomotor system (Figure 2F). This may induce abnormal behaviors that play the dream experience "dream enactment" as indicated by Morrison. ${ }^{63}$

\section{Sleep disturbances in Parkinson's disease}

Awake-sleep states are also impaired in PD. ${ }^{67,68}$ Sleep disturbances, such as daytime sleep attacks, nocturnal insomnia and RBD, are early signs of PD. ${ }^{69-71}$ Narcolepsy-like symptoms are also frequently observed. ${ }^{73}$ It has been also shown that PPN-ACh neurons are severely damaged in PD. ${ }^{73-75}$ Because the PPN has dense projections to the midbrain DA neurons, ${ }^{76,77}$ PPN-ACh neurons may affect awake-sleep states by modulating (1) DA systems projecting to the striatum, (2) muscle tone control systems, and (3) ARAS that affects the excitability of the forebrain.

RBD complicates various neurological disorders as described above. It is also frequent in PD and Alzheimer's disease where $\alpha$-synuclein appears. Because RBD often precedes to the onset of these diseases, the progression of Parkinson's disease proposed by Braak et al. ${ }^{6}$ may be also valid to describe the pathophysiology of RBD associated with neurodegenerative disorders. ${ }^{6}$ Degeneration of the cholinergic basal forebrain pathway to the cerebral cortex, limbic cortex and thalamus is involved in RBD in PD. ${ }^{78}$ In addition, frequency of RBD increases with DA replacement therapy using L-dopa. There is higher incidence in PD patients having gambling problem. ${ }^{79}$ Different from 5-HT, NA and histamine, DA neurons have higher firing rates during REM sleep. ${ }^{11}$ An activation of the limbic system caused by an increase in DA by the replacement therapy may also be involved in the development of this pathological condition. 


\section{Conclusion}

Under the "explicit consciousness", we put our volition or intention into actions (volitional behaviors) depending on behavioral context. The command signals generated in the cerebral cortex acts on the brainstem and spinal cord through dorsal forebrain system. On the other hand, alteration of "emotional consciousness" occurring at the sub cortical structures, particularly at the limbic system, activates the ventral forebrain system and elicits emotional behaviors that are accompanied by changes in autonomic function. Whether volitional or emotional, our behavior always requires an activation of hindbrain motor systems (Figure 1). That is, our behaviors can be expressed by appropriate functional linkage between the forebrain and hindbrain. ${ }^{4}$

Then, the question is how such a functional linkage is accomplished. We hypothesize that this functional linkage occurs based on Edelman's "Operation Theory" that it holds between systems with higher excitability. That is, a functional linkage occurs between more excitable forebrain system (either dorsal system or ventral system) and hindbrain system (either excitatory and locomotor systems or inhibitory system). As a result, expression of our behaviors is determined by the integration of the selected forebrain and hindbrain systems. Which system predominates in each of forebrain and hindbrain depends on the action of neurotransmitters such as monoamines, ACh and ORX in addition to excitatory and inhibitory inputs acting on these systems. Because key structures involved in the control of posture and locomotion exist in the midbrain, this is the place to link or switch functional connections between the forebrain and hindbrain systems.

\section{Acknowledgements}

This study was supported by JSPS KAKENHI Grant Numbers 26120004, 2529000, and AMED under Grant Number JP17ek0109078 to KT, and $15 \mathrm{~K} 06131$ and 26120006 to RC.

\section{Conflicts of interest}

The authors declared that there are no conflicts of interest.

\section{References}

1. Edelman G. Neural Darwinism. The Theory of Neuronal Group Selection. New York, USA: Basic Books; 1987.

2. Braun AR, Balkin TJ, Wesenten NJ, et al. Regional cerebral blood flow throughout the sleep-wake cycle. An H2 (15) O PET study. Brain 1997;120 (Pt 7):1173-1197.

3. Maquet P. Functional neuroimaging of normal human sleep by positron emission tomography. J Sleep Res. 2000;9(3):207-231.

4. Takakusaki K. Forebrain control of locomotor behaviors. Brain Research Review. 2008;57(1):192-198.

5. Takakusaki K. Neurophysiology of gait: from the spinal cord to the frontal lobe. Mov Disord. 2013;28(11):1483-1491.

6. Boeve BF, Silber MH, Saper CB, et al. Pathophysiology of REM sleep behaviour disorder and relevance to neurodegenerative disease. Brain 2007;130(Pt 11):2770-2788.

7. Nishino S, Okuro M, Kotorii N, et al. Hypocretin/orexin and narcolepsy: basic and clinical insights. Acta Physiol (Oxf). 2010;198(3):209-222.

8. Pillen S, Pizza F, Dhondt K, et al. Cataplexy and its mimics: clinical recognition and management. Curr Treat Options Neurol. 2017;19(6):23.
9. Grillner S, Georgopoulos AP, Jordan LM. Selection and initiation of motor behavior, in Neurons, Networks, and Motor Behavior. Stein PSG, et al. editors. Cambridge, USA: MIT Press; 1997. p. 3-19.

10. Jones BE. Activity, modulation and role of basal forebrain cholinergic neurons innervating the cerebral cortex. Prog Brain Res. 2004;145:157169.

11. Dahan L, Astier B, Vautrelle N, et al. Prominent burst firing of dopaminergic neurons in the ventral tegmental area during paradoxical sleep. Neuropsychopharmacology. 2007;32(6):1232-1241.

12. Koyama Y, Hayaishi O. Firing of neurons in the preoptic/anterior hypothalamic areas in rat: its possible involvement in slow wave sleep and paradoxical sleep. Neuroscience Research. 1994;19(1):31-38.

13. Brown RE, Basheer R, McKenna JT, et al. Control of sleep and wakefulness. Physiol Rev. 2012;92(3):1087-1187.

14. Stephenson-Jones M, Kardamakis AA, Robertson B, et al. Independent circuits in the basal ganglia for the evaluation and selection of actions Proc Natl Acad Sci USA. 2013;110(38):E3670-E3679.

15. Rossignol S. Neural control of stereotypic limb movements. Handbook of Physiology. Rowell LB, et al. editors. New York, USA: Oxford University Press; 1996. p. 173-216.

16. Mori S. Integration of posture and locomotion in acute decerebrate cats and in awake, free moving cats. Prog Neurobiol. 1987;28(2):161-196.

17. Grillner S. Control of locomotion in bipeds, tetrapods, and fish. In: Brooks VB, et al. editors. The Nervous System II. Bethesda, ML, USA: Am Physiol Soc Press; 1981. p. 1179-1236.

18. Takakusaki, Chiba R, Nozu T, et al. Brainstem control of locomotion and muscle tone with special reference to the role of the mesopontine tegmentum and medullary reticulospinal systems. J Neural Transm (Vienna). 2016;123(7):695-729.

19. Snijders AH, Takakusaki K, Debu B, et al. Physiology of freezing of gait Ann Neurol. 2016;80(5):644-659.

20. Takakusaki K. Functional neuroanatomy for posture and gait control. $J$ Mov Disord. 2017;10(1):1-17.

21. Grillner S, Robertson B, Stephenson-Jones M. The evolutionary origin of the vertebrate basal ganglia and its role in action selection. J Physiol. 2013;591(22):5425-543.

22. Alexander GE, Crutcher MD. Functional architecture of basal ganglia circuits: neural substrates of parallel processing. Trends Neurosci. 1990;13(7):267-271.

23. Takakusaki K, Takahashi K, Saitoh K, et al. Orexinergic projections to the cat midbrain mediate alternation of emotional behavioural states from locomotion to cataplexy. J Physiol. 2005;568(Pt 3):1003-1020.

24. Swanson LW, Mogenson GJ. Neural mechanisms for the functional coupling of autonomic, endocrine and somatomotor responses in adaptive behavior. Brain Research. 1981;228(1):1-34.

25. Lynd-Balta E, Haber SN. Primate striatonigral projections: a comparison of the sensorimotor-related striatum and the ventral striatum. $J$ Сотp Neurol. 1994;345(4):562-578.

26. Koob GF, Volkow ND. Neurocircuitry of addiction Neuropsychopharmacology. 2010;35(1):217-238.

27. Matsuyama K, Drew T. Organization of the projections from the pericruciate cortex to the pontomedullary brainstem of the cat: a study using the anterograde tracer Phaseolus vulgaris-leucoagglutinin. J Comp Neurol. 1997;389(4):617-641.

28. Keizer K, Kuypers HG. Distribution of corticospinal neurons with collaterals to the lower brain stem reticular formation in monkey (Macaca 
fascicularis). Exp Brain Res. 1989;74(2):311-318.

29. Aravamuthan BR, McNab JA, Miller KL, et al. Cortical and subcortical connections within the pedunculopontine nucleus of the primate Macaca mulatta determined using probabilistic diffusion tractography. J Clin Neurosci. 2009;16(3):413-420.

30. Jacobs JV, Lou JS, Kraakevik JA, et al. The supplementary motor area contributes to the timing of the anticipatory postural adjustment during step initiation in participants with and without Parkinson's disease. Neuroscience. 2009;164(2):877-885.

31. Kravitz DJ, Saleem KS, Baker CI, et al. A new neural framework for visuospatial processing. Nat Rev Neurosci. 2011;12(4):217-230.

32. Hikosaka O. GABAergic output of the basal ganglia. Prog Brain Res. 2007;160:209-226.

33. Swanson LW. Cerebral hemisphere regulation of motivated behaviour. Brain Res. 2000;886(1-2):113-164.

34. Alberico SL, Cassell MD, Narayanan NS. The vulnerable ventral tegmental area in Parkinson's disease. Basal Ganglia. 2015;5(2-3):5155 .

35. Fung SJ, Barnes CD. Membrane excitability changes in hindlimb motoneurons induced by stimulation of the locus coeruleus in cats. Brain Res. 1987;402(2):230-242.

36. Sakurai T. The neural circuit of orexin (hypocretin): maintaining sleep and wakefulness. Nat Rev Neurosci. 2007;8(3):171-181.

37. Schwartz MD, Kilduff TS. The Neurobiology of Sleep and Wakefulness. Psychiatr Clin North Am. 2015;38(4):615-644.

38. Mileykovskiy BY, Kiyashchenko LI, Siegel JM. Muscle tone facilitation and inhibition after orexin-a (hypocretin-1) microinjections into the medial medulla. J Neurophysiol. 2002;87(5):2480-2489.

39. Siegel JM. Hypocretin (orexn): Role in normal behavior and neuropathology. Annu Rev Psychol. 2004;55:125-148.

40. Takakusaki K, Kohyama J, Matsuyama K, et al. Medullary reticulospinal tract mediating the generalized motor inhibition in cats: parallel inhibitory mechanisms acting on motoneurons and on interneuronal transmission in reflex pathways. Neuroscience. 2001;103(2):511-527.

41. Takakusaki K, Kohyama J, Matsuyama K. Medullary reticulospinal tract mediating a generalized motor inhibition in cats: III. Functional organization of spinal interneurons in the lower lumbar segments. Neuroscience. 2003;121(3):731-146.

42. Takakusaki K, Takahashi M, Nakajima T, et al. Medullary reticulospinal tract mediating a generalized motor inhibition in cats: IV. Presynaptic control of primary afferents. Neuroscience.

43. Takakusaki K, Obara K, Nozu T, et al. Modulatory effects of the GABAergic basal ganglia neurons on the PPN and the muscle tone inhibitory system in cats. Arch Ital Biol. 2011;149(4):385-405.

44. Takakusaki K, Saitoh K, Harada H, et al. Evidence for a role of basal ganglia in the regulation of rapid eye movement sleep by electrical and chemical stimulation for the pedunculopontine tegmental nucleus and the substantia nigra pars reticulata in decerebrate cats. Neuroscience. 2004;124(1):207-220.

45. Takakusaki K, Habaguchi T, Ohtinata-Sugimoto J, et al. Basal ganglia efferents to the brainstem centers controlling postural muscle tone and locomotion: a new concept for understanding motor disorders in basal ganglia dysfunction. Neuroscience. 2003;119(1):293-308.

46. Takakusaki K, Kohyama J, Matsuyama K, et al. Synaptic mechanisms acting on lumbar motoneurons during postural augmentation induced by serotonin injection into the rostral pontine reticular formation in decerebrate cats. Exp Brain Res. 1993;93(3):471-482.
47. Takakusaki K, Shimoda N, Matsuyama K, et al. Discharge properties of medullary reticulospinal neurons during postural changes induced by intrapontine injections of carbachol, atropine and serotonin, and their functional linkages to hindlimb motoneurons in cats. Experimental Brain Research. 1994;99(3):361-374.

48. Leonard CS, Llinás R. Serotonergic and cholinergic inhibition of mesopontine cholinergic neurons controlling REM sleep: an in vitro electrophysiological study. Neuroscience. 1994;59(2):309-330.

49. Mileykovskiy BY, Kiyashchenko LI, Kodama T, et al. Activation of pontine and medullary motor inhibitory regions reduces discharge in neurons located in the locus coeruleus and the anatomical equivalent of the midbrain locomotor region. J Neurosci. 2000;20(22):8551-8558.

50. Jahn K, Deutschländer A, Stephan T, et al. Imaging human supraspinal locomotor centers in brainstem and cerebellum. Neuroimage. 2008;39(2):786-792.

51. Mori S, Matsui T, Kuze B, et al. Stimulation of a restricted region in the midline cerebellar white matter evokes coordinated quadrupedal locomotion in the decerebrate cat. J Neurophysiology. 1999;82(1):290 300 .

52. Mori S, Nakajima K, Mori F, et al. Integration of multiple motor segments for the elaboration of locomotion: role of the fastigial nucleus of the cerebellum. Prog Brain Res. 2004;143:341-351.

53. Sinnamon HM. Preoptic and hypothalamic neurons and initiation of locomotion in the anesthetized rat. Prog Neurobiol. 1993;41(3):323-344.

54. Takakusaki K. Modulation of the excitability of hindlimb motoneurons by the basal ganglia efferents to the brainstem in relation to the control of postural muscle tone and locomotion in the decerebrate cat. Society for Neuroscience. 2013;11749.

55. Takakusaki K, Tomita N, Yano M. Substrates for normal gait and pathophysiology of gait disturbances with respect to the basal ganglia dysfunction. J Neurology. 2008;255(Suppl 4):19-29.

56. Hikosaka O, Takikawa Y, Kawgoe R. Role of the basal ganglia in the control of purposive saccadic eye movements. Physiol Rev. 2000;80(3):954-978.

57. Takakusaki K. Auditory pathway in the brainstem contributes to the basal ganglia control of swallowing. Abstract 87th Meeting of the physiological society of Japan. Journal of Physiological Science. 2010;60(supplement):S-15.

58. Futami T, Takakusaki K, Kitai ST. Glutamatergic and cholinergic inputs from the pedunculopontine tegmental nucleus to dopamine neurons in the substantia nigra pars compacta. Neurosci Res. 1995;21(4):331-442.

59. Willie JT, Chemelli RM, Sinton CM, et al. Distinct narcolepsy syndromes in Orexin receptor-2 and Orexin null mice: molecular genetic dissection of Non-REM and REM sleep regulatory processes. Neuron. 2003;38(5):715-730.

60. Cvetkovic-Lopes V, Bayer L, Dorsaz S, et al. Elevated Tribbles homolog 2-specific antibody levels in narcolepsy patients. J Clin Invest. 2000;120(3):713-719.

61. Mahowald MW, Schenck CH. Insights from studying human sleep disorders. Nature. 2005;437:1279-1285.

62. Tsujino N, Tsunematsu T, Uchigashima M, et al. Chronic alterations in monoaminergic cells in the locus coeruleus in orexin neuron-ablated narcoleptic mice. PLoS One. 2013;8(7):e70012.

63. Morrison AR. A window on the sleeping brain. Scientific American. 1983;248(4):94-102.

64. Benarroch EE, Schmeichel AM, Parisi JE. Depletion of mesopontine cholinergic and sparing of raphe neurons in multiple system atrophy. Neurology. 2002;59(6):944-946. 
65. Arnulf I, Merino-Andreu M, Bloch F, et al. REM sleep behavior disorder and REM sleep without atonia in patients with progressive supranuclear palsy. Sleep. 2005;28(3):349-354.

66. Ringman JM, Simmons JH. Treatment of REM sleep behavior disorder with donepezil: a report of three cases. Neurology. 2000;55(6):870-871.

67. Bliwise DL, Willians ML, Irbe $\mathrm{D}$, et al. Inter-rater reliability for identification of REM sleep in Parkinson's disease. Sleep. 2000;23(1):671-676.

68. Eisensehr I, Lindeiner H, Jager M, et al. REM sleep behavior disorder in sleep-disordered patients with versus without Parkinson's disease: is there a need for polysomnography? J Neurol Sci. 2001;186(1-2):7-11.

69. Askenasy JJ. Approaching disturbed sleep in late Parkinson's disease: first step toward a proposal for a revised UPDRS. Parkinsonism Relat Disord. 2001;8(2):123-131.

70. Ferini-Strambi L, Zucconi M. REM sleep behavior disorder. Clinical Neurophysiology. 2000;111(Supplement 2):S136-140.

71. Larsen JP, Tandberg E. Sleep disorders in patients with Parkinson's disease: epidemiology and management. CNS Drugs. 2001;15(4):267275 .

72. Haq IZ, Naidu Y, Reddy P, Chaudhuri KR. Narcolepsy in Parkinson's disease. Expert Rev Neurother. 2010;10(6):879-884.

73. Hirsch EC, Graybiel AM, Duyckaerts C, et al. Neuronal loss in the pedunculopontine tegmental nucleus in Parkinson disease and in progressive supranuclear palsy. Proc Natl Acad Sci USA. 1987;84(16):5976-5980.
74. Jellinger K. The pedunculopontine nucleus in Parkinson's disease, progressive supranuclear palsy and Alzheimer's disease. Journal of Neurology, Neurosurgery and Psychiatry. 1988;51(4):540-543.

75. Zweig RM, Jankel WR, Hedreen JC, et al. The pedunculopontine nucleus in Parkinson's disease. Ann Neurol. 1989;26(1):41-46.

76. Mena-Segovia J, Winn P, Bolam JP. Cholinergic modulation of midbrain dopaminergic systems. Brain Res Rev. 2008;58(2):265-271.

77. Takakusaki K, Shiroyama T, Yamamoto T, et al. Cholinergic and noncholinergic tegmental pedunculopontine projection neurons in rats revealed by intracellular labeling. J Comp Neurol. 1996;371(3):345-361.

78. Kotagal V, Albin RL, Müller ML, et al. Symptoms of rapid eye movement sleep behavior disorder are associated with cholinergic denervation in Parkinson disease. Ann Neurol. 2012;71(4):560-568.

79. Peever J, Luppi PH, Montplaisir J. Breakdown in REM sleep circuitry underlies REM sleep behavior disorder. Trends Neurosci. 2014;37(5):279-288. 